\title{
Sacrococcygeal Teratoma in a Singleton Male Instance of Cornelia de Lange Syndrome: A Case Report
}

QR CODE

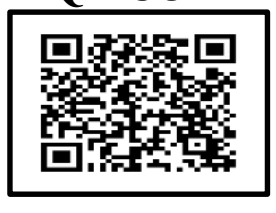

\section{SEYED REZA SAMSAMSHARIAT}

Sacrococcygeal teratoma, sirenomelia, VATER association, anencephaly and holoprocencephaly are occasionally observed in patients with Cornelia de Lange syndrome (CdLS; OMIM 122470). Here, I present an exceptional case of a non-twin, singleton newborn with CdLs who also had a sacrococcygeal teratoma.

KEYWORDS: Sacrococcygeal Teratoma, Cornelia De Lange Syndrome, VATER Association.

\section{INTRODUCTION}

Background Cornelia de Lange syndrome (CdLS; OMIM 122470) is a dominantly inherited, multisystem-developmental disorder characterized by both growth and cognitive retardation, abnormalities of the upper limbs, gastroesophageal dysfunction, cardiac, ophthalmologic and genitourinary anomalies, hirsutism and characteristic facial features. ${ }^{1-3}$ Genital anomalies, pyloric stenosis, congenital diaphragmatic hernias, cardiac septal defects, hearing loss and autistic and self-injurious tendencies also frequently occur. ${ }^{2}$ Prevalence is estimated to be as high as 1 in 10,00o. ${ }^{4}$ A one-hour old neonate who had died in the Neonatal Intensive Care Unit of a local hospital was referred to my laboratory to undergo a diagnostic autopsy. Structural defects occur in some cases of CdLs.

These are commonly seen in live-born, monozygotic twins with this syndrome. Among monochorionic, monoamniotic and monozygotic twins, malformations are most common. The defects seen with increased frequency in monozygotic twins include sacrococcygeal teratoma, sirenomelia, VATER association, anencephaly and holoprocencephaly. ${ }^{5}$ Noteworthy in this case however is the fact that the affected fetus was a singleton and not a twin. Although Cornelia de Lange syndrome is caused by mutations in NIPBL, ${ }^{6}$ partial duplication of chromosome 39 correlated with sacrococcygeal teratoma has been suggested as the cause of CdLs in exceptional cases. ${ }^{7}$

Most individuals with typical CdLS have de novo heterozygous loss-of-function mutations in NIPBL, and a significant sub-group of these patients represent cases of the syndrome due to post-mitotic mosaicism.
Less typical CdLs variants are found to have mutations in other cohesion components such as $\mathrm{SMC}_{1} \mathrm{~A}, \mathrm{SMC}_{3}$, HDAC8 and RAD218.

\section{CASE PRESENTATION}

The cadaver of this case belonged to a one-hour old male, first-born neonate to non-consanguineous parents, a 22-year old mother and a 30-year old father, with no family history of genetic disorders on either side. The mother denied spotting, diabetes, or UTI during her pregnancy, but was found to have mild hypertension and proteinuria. Results of routine screens were within normal limits, but sonography indicated several abnormalities such as pyelectasis, megacystis, oligohydramnios and clubfoot (Figures 1 and 2). The more recent sonograph showed bilateral hydronephrosis and edema in the lower limbs and, consequently, the mother was offered with the choice to induce abortion, but she declined. Due to exacerbation of her hypertension in the $32^{\text {nd }}$ week of the gestation, the fetus was delivered by cesarean section. He was alive for 40 minutes in the ICU, but then expired.

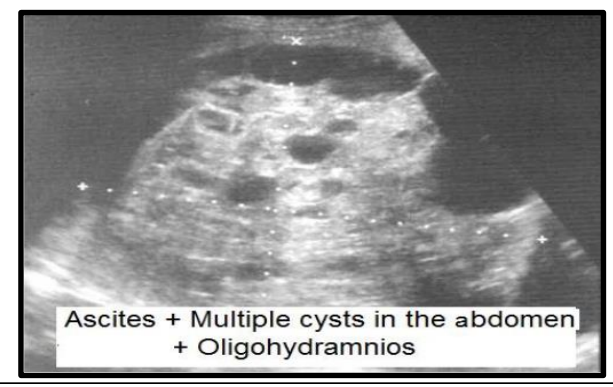

Figure 1. Sonography of the neonate showing ascites, multiple cysts in the abdomen and oligohydraminos. 


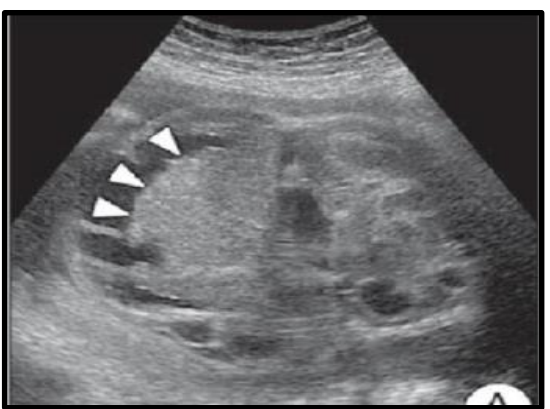

Figure 2. Axial US demonstrating heterogeneous image in the pelvis, with predominantly solid.

\section{AUTOPSY FINDINGS}

The fetal body was that of a male. It had a crown-heel height of $42 \mathrm{~cm}$ and a crown-rump height of $26 \mathrm{~cm}$. The lower limbs were $16 \mathrm{~cm}$ long and the head circumference was $34 \mathrm{~cm}$. No bruising was observed, but some maceration of the trunk. There was 2+ edema over the abdomen and the lower limbs. 1+ cyanosis was seen but no dehydration. Craniosynostosis of the skull was present. The eyes were deeply set and had epicanthal folds. The nose was small with a depressed root and upward tip (Figure 3). The philtrum was long. The ears had minimal dysplasia. The mouth and chain were small. The neck was normal. There was abdominal enlargement and a bulging sacral mass (Figure 4). The hands had camptodactyly and clinodactyly. The lower limbs were edematous with folded skin (Figure 4). The fetus had club feet. The umbilical cord was $4.5 \mathrm{~cm}$ long, $1.6 \mathrm{~cm}$ wide and was found to contain three blood vessels in cross-section.

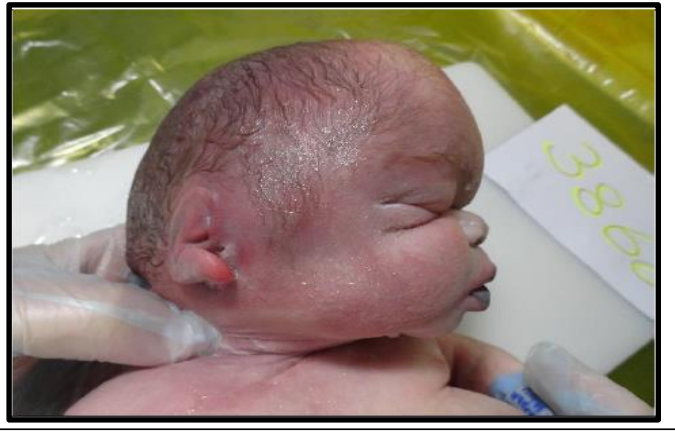

Figure 3. Small nose with depressed root and upward tip, long philtrum and somewhat dysplastic ears.

On dissection, the thoracic wall was neither atrophic nor emphysematous. The right and left part of the diaphragm were intact. No pneumothorax was observed, however sever bilateral hydrothorax was present with clear yellow-tinged fluid. The thymus measured $4.5 \times 3 \times 1.5 \mathrm{~cm}$. The heart was cone shaped measuring $4.5 \times 4 \times 3 \mathrm{~cm}$.

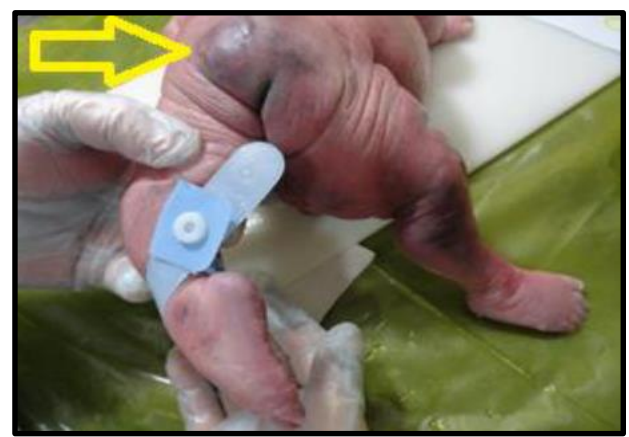

Figure 4. Bulging sacral mass (yellow arrow) and lower limb edema with folded skin and Club feet.

The chambers and large vessels were within normal limits. The pericardium was free of attachments and its fluid volume appeared above normal limits. Bronchi and esophagus were within normal limits. Each lung was $6.5 \times 3 \times 2 \mathrm{~cm}$ and were both congested and airless. No ascites were observed in the abdominal cavity but there was a large tumor under the liver occupying the abdominal cavity. It measured $12 \mathrm{~cm}$ in diameter and its peduncle was attached to the sacral portion of the pelvis (Figure 5). The liver was soft and autolytic measuring $13.5 \times 9 \times 4 \mathrm{~cm}$. The intestines were laterally displaced. The stomach measured $4 \times 1.5 \times 0.7$ $\mathrm{cm}$ and its wall was $0.1 \mathrm{~cm}$ thick. The spleen was beanshaped measuring $4.8 \times 3.5 \times 1.6 \mathrm{~cm}$. The pancreas measured $2.3 \times 0.6 \times 0.4 \mathrm{~cm}$. Both ureters were $0.2 \mathrm{~cm}$ in diameter. Both kidneys measured $6 \times 3 \times 2.5 \mathrm{~cm}$ with a cortex $0.2 \mathrm{~cm}$ thick and were autolytic. The adrenals each measured $2.5 \times 2.5 \times 1 \mathrm{~cm}$ and showed hemorrhage in the centers. The bladder's diameter measured $4 \mathrm{~cm}$ and it contained clear urine. Its wall had a thickness of $0.2 \mathrm{~cm}$. Both testis measured $1.3 \times 0.6 \times 0.3 \mathrm{~cm}$ and were in the abdominal cavity, undescended. The genitals were of the male type. The proximal part of the penis had an erosion and was hypoplastic ${ }^{9}$. The anus was normal.

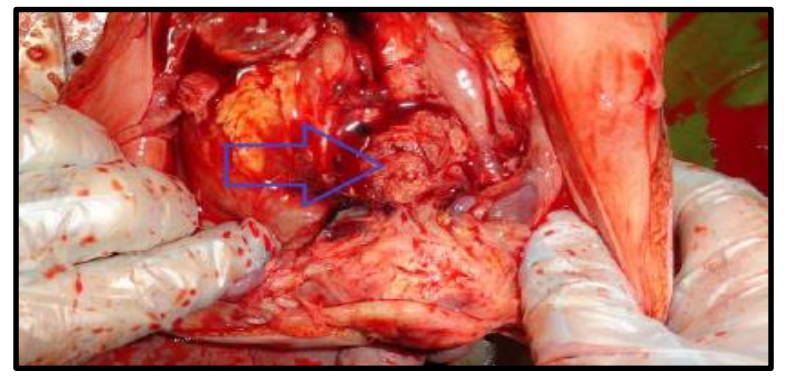

Figure 5. The tumor peduncle was attached to the sacral portion of the pelvis (arrow). 
Microscopic examination of the tissue samples from the abdominal mass revealed various, welldifferentiated representatives of all three embryonic tissue layers, including neuroectodermal. Solid and cystic foci of epithelium were admixed with glandular or tubular structures. These mixed foci were separated by mesenchymal proliferation. Both glial and neuronal tissues were observed as well as cartilage (Figure 6).

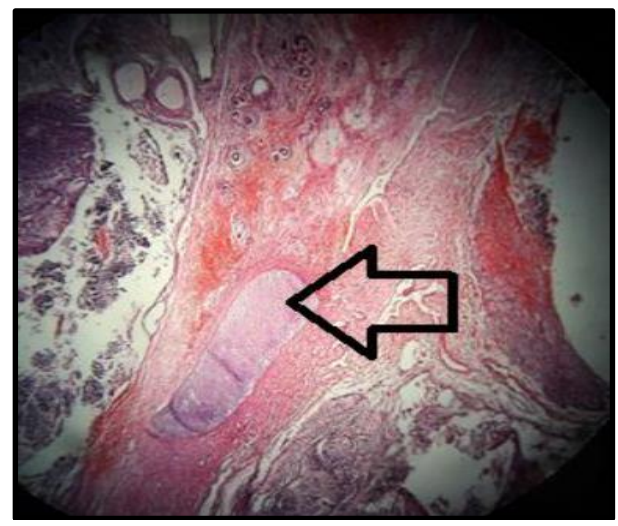

Figure 6. All three embryonic tissue layers were represented including neuroectodermal. Solid and cystic foci of epithelium were admixed with glandular or tubular structures. Glial and neuronal tissue was seen as well as cartilage (arrow).

\section{DISCUSSION}

Although most prior investigations of Cornelia de Lange's syndrome have reported a prevalence of 1 in $10,000,{ }^{4}$ data from Denmark show an incidence of $1 / 200,0^{10} 0^{10}$ with a total of 24 patients reported, 10 males and 14 females.

A variety of mutations are responsible for the clinical syndrome known as $\mathrm{CdLs},{ }^{6,7}$ but cases with sacrococcygeal teratoma are correlated with a partial duplication of chromosome $39^{7}$. The unusual case reported here of a singleton neonate likely belongs to this latter group.

\section{REFERENCES}

1. de Lange $C$. Sur Un type nouveau de dégénératio (typus Amstelodamensis). Arch Méd Enfants. 1993;36:713-9.

2. Jackson L, Kline AD, Barr MA, Koch S. de Lange syndrome: a clinical review of 310 individuals. Am J Med Genet. 1993;47:940-6.

3. Ireland M, Donnai D, Burn J. Brachmann-de Lange syndrome. Delineation of the clinical phenotype. Am. J Med Genet. 1993;47:959-4.

4. Opitz JM. The Brachmann-de Lange syndrome. Am. J. Med. Genet. 1985;22:89-102.

5. Sheela, SR. Cornelia de Lange syndrome: Discordance in twins." Indian paediatrics 1999; 36(12):1267-9.

6. Krantz ID, McCallum J, DeScipio C, Kaur M, Gillis LA, Yaeger D. Cornelia de Lange syndrome is caused by mutations in NIPBL, the human homolog of Drosophila melanogaster Nipped-B. Nature genetics 2004;36(6):631-5.

7. Dundar M, Uzak A, Erdogan M, et al. Partial trisomy 39 in a child with sacrococcygeal teratoma and Cornelia de Lange syndrome phenotype. Genet Couns. 2011;22:199-205.

8. Ansari M, Poke G, Ferry Q, Williamson K, Aldridge $\mathrm{R}$, Meynert $\mathrm{AM}$, et al. Genetic heterogeneity in Cornelia de Lange syndrome (CdLS) and CdLS-like phenotypes with observed and predicted levels of mosaicism." Journal of Medical Genetics, 2014;51(10):659-68.

9. Sheela SR. Cornelia de Lange syndrome: Discordance in twins. Indian pediatrics 1999;36(12):1267-9.

10. Beck B. Epidemiology of Cornelia de Lange's syndrome. Acta Paediatrica 1976;65(4):631-38. 